\title{
Trans-Perineal Sonographic Findings of Anal Sphincter Muscle Complex in Imperforated Anus: Diagnostic Pitfalls
}

\author{
Seyed Ali Alamdaran (iD ${ }^{1,}{ }^{*}$, Ali Abdollahi ${ }^{2}$, Ali Feyzi ${ }^{2}$, Farideh Jamali-Behnam ${ }^{3}$, Mehdi Yousefzadeh \\ Talfavani ${ }^{4}$, Mehdi Fathi ${ }^{5}$, Ali Azadmand ${ }^{4}$, Marjan Joudi ${ }^{4}$, Mehdi Parvizi Mashhadi ${ }^{6}$, Maryam \\ Tayebi-Meybodi ${ }^{7}$ and Alireza Sabzevari ${ }^{5}$ \\ ${ }^{1}$ Department of Radiology, Faculty of Medicine, Mashhad University of Medical Sciences, Mashhad, Iran \\ ${ }^{2}$ Department of Radiology, Mashhad University of Medical Sciences, Mashhad, Iran \\ ${ }^{3}$ Surgical Oncology Research Center, Mashhad University of Medical Sciences, Mashhad, Iran \\ ${ }^{4}$ Department of Surgery, Mashhad University of Medical Sciences, Mashhad, Iran \\ ${ }^{5}$ Department of Anesthesiology, Mashhad University of Medical Sciences, Mashhad, Iran \\ ${ }^{6}$ Department of Pediatric Surgery, Faculty of Medicine, Mashhad University of Medical Sciences, Mashhad, Iran \\ ${ }^{7}$ Department of Emergency Medicine, Mashhad University of Medical Sciences, Mashhad, Iran \\ "Corresponding author: Department of Radiology, Faculty of Medicine, Mashhad University of Medical Sciences, Mashhad, Iran. Email: alamdarana@mums.ac.ir
}

Received 2020 January 18; Revised 2020 July 29; Accepted 2020 August 03.

Keywords: Ultrasound, Imperforate Anus (IA), Child

\section{Introduction}

The exact anus reconstruction from the middle of the sphincter complex is critical in patients with imperforated anus for continence preservation. In this regard, we designed a study with the purpose to investigate the ability of ultrasound in detecting the sphincter muscle complex in children with imperforated anus. This study entitled: "Preoperative Trans-Perineal Sonographic Findings in Children with Imperforated Anus for Detection of Anal Sphincter Muscle Complex and the Anal Canal Pathway: A Pilot Study" by Alamdaran et al. (1) was published in the Iran J Radiol. 2018; 15 (3). In this study, transperineal ultrasound findings were correlated with muscle stimulator and intraoperative findings in 25 patients. We hereby add this commentary to it.

\section{Argument}

As we noted, we have completed the study entitled "Preoperative Trans-Perineal Sonographic Findings in Children with Imperforate Anus for Detection of Anal Sphincter Muscle Complex and the Anal Canal Pathway: A Pilot Study" by Alamdaran et al. (1) published in the Iran J Radiol. 2018; 15 (3).

The exact anus reconstruction from the middle of the sphincter complex is critical in patients with imperforated anus for continence preservation. We designed this study to investigate the ability of ultrasound in identifying anal sphincter muscle complex in infants with Imperforated anus.

This cross-sectional study was performed at Mashhad University of Medical Sciences from 2016 to 2018, after approval by Ethic Committee of university and obtaining informed consent from the parents. Twenty-five patients ( 2 days until 12 week age, 22 boys and three girls) with imperforated anus were selected, of whom 21 had previous colostomy and four cases were newborn. Pre-operative transperineal ultrasound (SonoSite Model S-Nerve with a 12 $\mathrm{MHz}$ linear probe) was performed in lithotomy position in both sagittal and coronal planes. For better visualization of the rectal pouch, a Foley catheter was fixed inside the distal limb of colostomy and normal saline was injected in the rectum. Then, ultrasound findings were correlated with distal colostogram (Figure 1), muscle stimulator and intraoperative findings. Only four cases of our patients were the low type and the others were the high type.

\subsection{Ultrasound Findings}

The new ultrasound findings in this study that have not been mentioned in other studies include (1) concentric multi-layered structures in the subcutaneous tissues (probably anal pit); (2) concentric multi-layered structures in the deep pelvic floor (probably anal sphincter complex); (3) difference in distance of rectal pouch and the surface of 

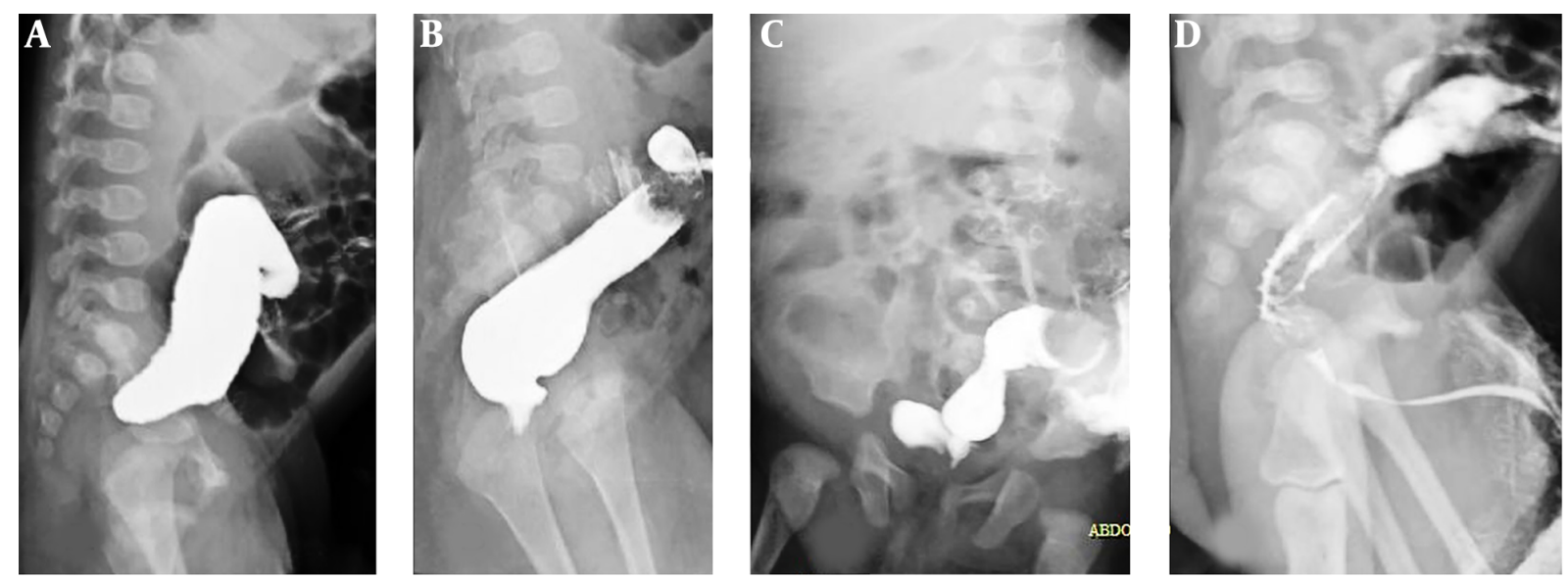

Figure 1. Distal colostogram view of patients; A, High type of IA without fistula; B, Low type of IA without fistula; C, High type of IA with bladder neck fistula; D, High type of IA with recto-bulbar fistula.

skin through probably anal pit and anal sphincter complex pathway with the shortest distant; (4) the ability of sonography to differentiate different types of internal fistula.

1) The concentric multi-layered structures in the subcutaneous and deep pelvic floor have gut signature appearance. These two concentric multi-layered structures were mainly visible in the coronal view and may not be seen in the sagittal plane (Figure 2).

Superficial structure (probably anal pit) has a transverse diameter of $1.8-10 \mathrm{~mm}$ (mean $=4 \mathrm{~mm}$ ) and a depth of $4-10 \mathrm{~mm}$ (mean $=5.4 \mathrm{~mm}$ ). This structure was mainly seen in the coronal plane and it is invisible in 16\% (4 of 25) of the patients. The muscle stimulator and intra-operative findings show that it was correlated with the superficial part (striated) of muscle complex sphincter. This finding may have a prognostic value for continence.

The deep concentric multi-layered structure was seen in all patients with the mean thickness of $8.1 \mathrm{~mm}$ (range, $3.2-13 \mathrm{~mm}$ ) and occasionally asymmetric and eccentric. It could be related to the smooth part of muscle complex sphincter or levator ani muscle that needs future researches.

2) The alignment of the center of these two concentric multi-layered structures in the subcutaneous and deep pelvic floor is straight or curved, and occasionally not aligned.

3) The mean of the shortest measurable distance between the rectal pouch and the surface of the skin was 13.6 $\mathrm{mm}$ (range, 2.5-28 $\mathrm{mm}$ ) and probably through anal sphincter complex pathway it was $16.5 \mathrm{~mm}$ (range, 3-28 mm) with 0-9 mm difference (Figure 3A).

4) Ultrasound not only detects internal fistula in all patients $(2,3)$, but also differentiates its subtypes [bulbar (6), bladder neck (4), mid-prostatic (4), perineal (2), rectomembranous (1) and vestibular] in the sagittal plane especially after saline infusion through distal colostomy; (1) (Figure 3B-D). Fistula was not seen in seven cases. The limitation of ultrasound was the exact differentiation of the three mid-prostatic, perineal and recto-membranous types from each other.

5) Fistula was not seen in seven cases. The limitation of ultrasound was the exact differentiation of the three mid-prostatic, perineal and recto-membranous types from each other.

\subsection{The Diagnostic Pitfalls}

However, in spite of the mentioned points, we found four sonographic diagnostic pitfalls in these patients:

1) Iso-echogenicity of the muscle sphincter complex with perineal fat in the new born: Although in the adult, hypoechoic muscles are completely differentiable from echogenic fat, in the infant, iso-echogenicity of the muscle sphincter complex with perineal fat is problematic and questionable. In addition, decreasing resolution of ultrasound images with the increase in depth cause poor visualization of the deep muscle sphincter. Therefore, differentiation of the muscle complex sphincter from other tissues is difficult by ultrasound, especially in high type (Figure $3 \mathrm{~A}$ and $\mathrm{B})$.

2) Similarity of muscle sphincter complex with ectopic rectal pouch in perinea: Ectopic rectal pouch of the high type IA with perineal fistula mimic the gut signature appearance of concentric multi-layered structures and may 

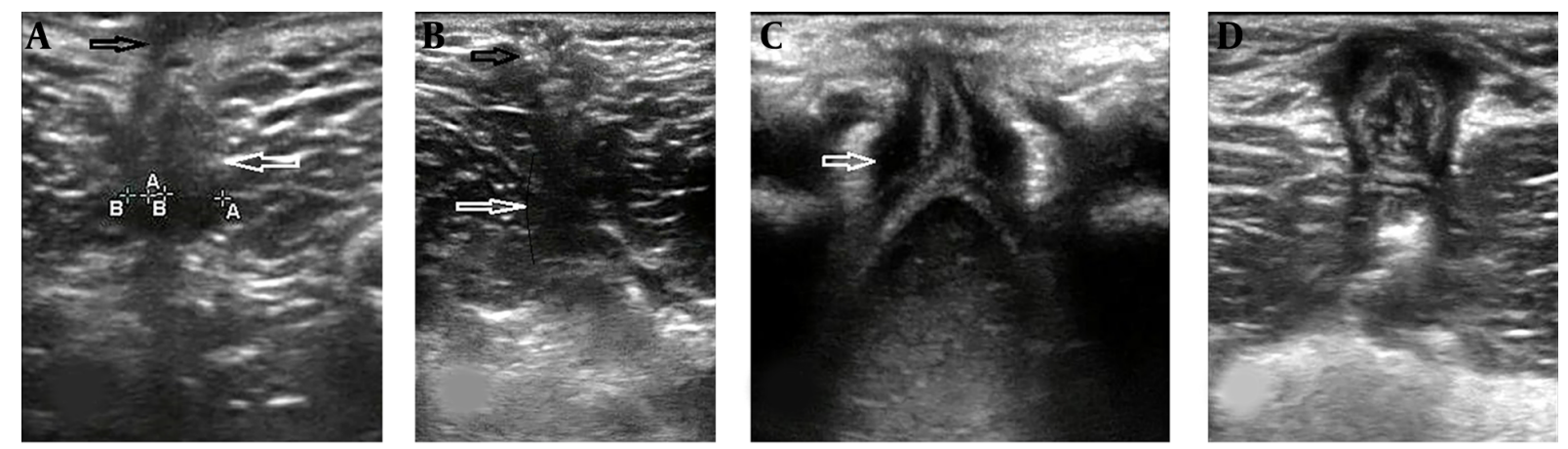

Figure 2. The appearance and pitfalls of two concentric multi-layered structures; A, Isoechoic deep muscle sphincter with perineal fat; B, Poor visualization of deep muscle sphincter complex in the high type of imperforate anus; C and D, Similarity of low type of imperforate anus (C) with ectopic rectal pouch of the high type IA with perineal fistula (D) that mimic the low type of imperforate anus. The anal pit is demarcated with black arrows and the deep muscle sphincter is demarcated with white arrows.
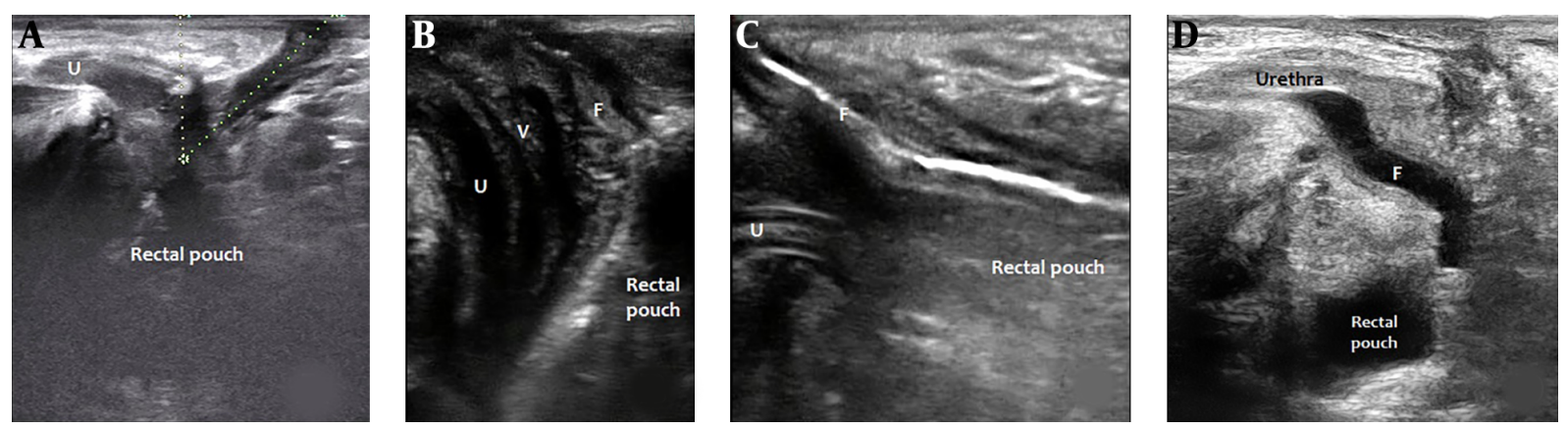

Figure 3. This image shows the difference between the rectal pouch and the surface of the skin through the anal sphincter complex pathway with the shortest distance (A). Different types of internal fistula; rectovestibular (B), rectoperineal (C), rectobulbar (D) during saline infusion.

be wrongly mistaken with the low type of anomaly (Figure 3 C and D).

3) Various cut offs for distance between rectal pouch and the surface of skin: In literature, there are some diagnostic cut off overlaps from $5 \mathrm{~mm}$ until $25 \mathrm{~mm}$ to determine the level of anorectal malformations (low and high). In addition, our results show the measurement method (the shortest or through anal sphincter complex pathway) is associated with different distances (2-4).

4) The surgical distance is longer than the ultrasound distance: the mean distance between the rectal pouch and the surface of the skin was $16.8 \pm 0.8$ in ultrasound and 29.1 $\mathrm{mm}$ in surgery with significant difference $(\mathrm{P}=0.001)$. Pressure of ultrasound probe or saline infusion through distal colostomy causes underestimation of distance between rectal pouch and the surface of skin.

Overall, although these ultrasound findings could help to determine the level and type of disorder in order to plan preoperatively and select the less invasive surgical technique, and even maybe image guided surgery (5), it has the number of diagnostic pitfalls that could lead to serious diagnostic errors.

Today, ultrasound is the most available diagnostic modality, but there are limited numbers of research that have been performed to determine the level of anorectal malformations. For better understanding of these sonographic findings and anatomic correlations, further research, especially sonographic - anatomic correlation during surgery is necessary.

\section{Conclusion}

Trans-perineal ultrasound in imperforated anus can diagnose internal fistula and all types of it especially after saline infusion through distal colostomy. This resolves the need to perform distal colostomy. However, it has a number of diagnostic pitfalls especially in determining the deep muscle sphincter complex. 


\section{Acknowledgments}

The authors gratefully acknowledge the contribution of the surgeons and members of operation room of Akbar and Dr. Sheikh hospitals in supporting us to conduct the research.

\section{Footnotes}

Authors' Contributions: Seyed Ali Alamdaran, Marjan Joudi, and Mehdi Parvizi Mashhadi carried out the study design, participated in data collection and drafted the manuscript. Seyed Ali Alamdaran and Mehdi Parvizi Mashhadi participated in the design of the study and performed the statistical analysis. Seyed Ali Alamdaran and Marjan Joudi conceived the study, participated in its design and coordination and performed the interpretation of data. All authors read and approved the final version.

Conflict of Interests: The authors have no conflicts of interest to declare.

Funding/Support: This study was supported in part by Dr. Alamdaran's grant from the deputy for research of Mashhad University of Medical Sciences.

\section{References}

1. Alamdaran SA, Joudi M, Azadmand A, Sabzevari A, Abdollahi A, Feyzi A, et al. Preoperative Trans-Perineal Sonographic Findings in Children with Imperforate Anus for Detection of Anal Sphincter Muscle Complex and the Anal Canal Pathway: A Pilot Study. Iran J Radiol.2018;15(3) doi: 10.5812/iranjradiol.57944.

2. Choi YH, Kim IO, Cheon JE, Kim WS, Yeon KM. Imperforate anus: determination of type using transperineal ultrasonography. Korean J Radiol. 2009;10(4):355-60. doi: 10.3348/kjr.2009.10.4.355. [PubMed: 19568463]. [PubMed Central: PMC2702044].

3. Ekwunife $\mathrm{OH}$, Umeh EO, Ugwu JO, Ebubedike UR, Okoli CC, Modekwe VI, et al. Comparison of trans-perineal ultrasound-guided pressure augmented saline colostomy distension study and conventional contrast radiographic colostography in children with anorectal malformation. AfrJPaediatr Surg. 2016;13(1):26-31. doi: 10.4103/01896725.181703. [PubMed: 27251520]. [PubMed Central: PMC4955457].

4. Haber HP, Seitz G, Warmann SW, Fuchs J. Transperineal sonography for determination of the type of imperforate anus. AJR Am J Roentgenol. 2007;189(6):1525-9. doi: 10.2214/AJR.07.2468. [PubMed: 18029895].

5. Alamdaran SA, Hashemi J, Feyzi A, Azadmand A, Mahdavi Rashed M, Jafari S, et al. Ultrasound Guided Wire Localization of Anal Tract in Imperforate Anus. Iran J Pediatr. 2018;28(4). doi: 10.5812/ijp.65338. 